\title{
Updates in advanced prostate cancer 2018
}

\author{
Andrew J. Armstrong (1) ${ }^{1}$
}

Received: 20 September 2018 / Accepted: 20 September 2018 / Published online: 2 October 2018

C) Springer Nature Limited 2018

In 2017-18, several notable advances were made in the care of men with advanced or metastatic prostate cancer, and Prostate Cancer and Prostatic Diseases provided important insights and perspectives around each of these major advances. As one among the top global prostate-specific journals, we are seeing a major increase in our impact factor and global reach, paralelling the increase in the basic understanding of the biology and treatment of prostate cancer. In this editorial, I have selected nine key manucripts from the past year in our journal that highlight and provide novel insights into these important clinical and translational advances. These are provided below with their citations, links, and a brief description of the article's importance, with the intent of bringing attention to these works to the prostate cancer research community. These are in no particular order, as each has been heavily cited and read, and emphasizes the unique aspects of advanced prostate cancer clinical practice and research.

1. Non-metastatic castration resistant prostate cancer: a review of current and emerging medical therapies [1]. In 2018, both enzalutamide and apalutamide became USFDA approved in men with non-metastatic castration-resistant prostate cancer (nmCRPC) based on a potential surrogate end point of metastasis-free survival. This review covers these new data and emerging therapies in this unique disease state, and the particular challenges of drug development in this setting.

2. Addition of abiraterone, docetaxel, bisphosphonate, celecoxib or combinations to androgendeprivation therapy (ADT) for metastatic

Andrew J. Armstrong

andrew.armstrong@duke.edu

1 Duke Cancer Institute, Divisions of Medical Oncology and Urology, Duke University, DUMC Box 103861, Durham, NC 27710, USA hormone-sensitive prostate cancer (mHSPC): a network meta-analysis [2]. In the June of 2017, the treatment of men with metastatic hormone-sensitive prostate cancer (mHSPC) was transformed by results from the STAMPEDE and LATTITUDE trials showing improved survival with abiraterone, and previously from CHAARTED and STAMPEDE with docetaxel in addition to androgen deprivation therapy. This meta-analysis looks at all mHSPC trials and suggests that abiraterone may have superior efficacy to docetaxel in these men.

3. Can RECIST response predict success in phase 3 trials in men with metastatic castration-resistant prostate cancer? [3] A major issue in drug development in advanced prostate cancer remains the lack of surrogate intermediate end points for overall survival, which can be quite prolonged in many men. This original study examined all phase- 2 and -3 trials of men with mCRPC to look at whether significant measurable disease response rates by RECIST in phase-2 trials led to positive phase-3 trials, and suggests that this may be the case for certain single agents depending on the mechanism of action of the specific therapy.

4. Development of comorbidities in men with prostate cancer treated with androgen deprivation therapy: an Australian population-based cohort study [4]. A major issue facing men with advanced prostate cancer is that of treatment-related toxicities impacting their survivorship, particularly androgen deprivation therapy (ADT). This study describes the risks of secondary metabolic and cardiovascular morbidities due to ADT at the national level using a large national database.

5. p53 status in the primary tumor predicts efficacy of subsequent abiraterone and enzalutamide in castration-resistant prostate cancer [5]. A major unmet need in the treatment of men with mCRPC is that of predictive biomarkers that can capture the heterogeneity of outcomes with specific therapies. This article finds that men with p53 mutant mCRPC, 
as identified in the primary tumor, have worse outcomes when treated with enzalutamide or abiraterone, and, similar to AR-V7, represents a group with a poor prognosis and unmet need for novel therapies.

6. Current approaches to incorporation of radium223 in clinical practice [6]. In 2017-18, the world of radium-223 was turned upside down with the release of early data from the ERA-223 study suggesting more fractures and worse outcomes with abiraterone acetate and radium-223 as compared to abiraterone alone in men with mCRPC in the first-line setting. This review updates us on where radium-223 may best fit into the current treatment paradigm and ongoing studies that will help to clarify combination approaches.

7. Platinum sensitivity in metastatic prostate cancer: does histology matter? [7]. Treatment-emergent neuroendocrine or small-cell prostate cancer (NEPC) has become a major issue with the earlier use of novel potent AR-targeted therapies such as abiraterone and enzalutamide. Interestingly, recent data suggest that NEPC may be disconnected from the presence of DNA repair defects, which have been associated with PARP and platinum sensitivity [8]. This analysis found that platinum sensitivity in men with mCRPC was not associated with neuroendocrine histology, affirming the need for more specific and genomic predictors of platinum and PARP inhibitor efficacy beyond simple histology.

8. The use of PET/CT in prostate cancer [9]. The imaging and staging of recurrent prostate cancer continues to evolve with the development of novel PET tracers, including PSMA, fluciclovine, choline, and sodium fluoride, among others. This review discusses the pros and cons and evidence supporting each of these modalities, and suggests ways forward for evidence generation to support their more routine use for initial staging and restaging for recurrent disease after local therapy, or for metastatic evaluation and response to systemic therapies.

9. Imaging response during therapy with radium-223 for castration-resistant prostate cancer with bone metastases-analysis of an international multicenter database [10]. This retrospective analysis provides valuable data on how mCRPC patients progress, particularly in soft tissue sites, while on radium-223.

10. Lutetium-177-labelled anti-prostate-specific membrane antigen antibody and ligands for the treatment of metastatic castrate-resistant prostate cancer: a systematic review and meta-analysis [11]. This review discusses novel PSMA tracers for imaging and radioimmunotherapy of men with recurrent prostate cancer, now in phase- 3 trials.

Together, these works encompass advances in precision medicine, imaging, and biomarkers in advanced prostate cancer, as well as in the survivorship of these men. These selected works take the field closer to the goal of delivering such optimal care to our patients.

\section{References}

1. Alpajaro SIR, Harris JAK, Evans CP. Non-metastatic castration resistant prostate cancer: a review of current and emerging medical therapies. Prostate Cancer Prostatic Dis. 2018. https://doi.org/ 10.1038/s41391-018-0078-1.

2. Tan PS, Aguiar P Jr, Haaland B, Lopes G. Addition of abiraterone, docetaxel, bisphosphonate, celecoxib or combinations to androgen-deprivation therapy (ADT) for metastatic hormonesensitive prostate cancer (mHSPC): a network meta-analysis. Prostate Cancer Prostatic Dis. 2018. https://doi.org/10.1038/ s41391-018-0055-8.

3. Brown LC, Sonpavde G, Armstrong AJ. Can RECIST response predict success in phase 3 trials in men with metastatic castrationresistant prostate cancer? Prostate Cancer Prostatic Dis. 2018;21:419-30.

4. Ng HS, Koczwara B, Roder D, Vitry A. Development of comorbidities in men with prostate cancer treated with androgen deprivation therapy: an Australian population-based cohort study. Prostate Cancer Prostatic Dis. 2018. https://doi.org/10.1038/ s41391-018-0036-y.

5. Maughan BL, Guedes LB, Boucher K, Rajoria G, Liu Z, Klimek $\mathrm{S}$, et al. p53 status in the primary tumor predicts efficacy of subsequent abiraterone and enzalutamide in castration-resistant prostate cancer. Prostate Cancer Prostatic Dis. 2018;21:260-8.

6. Parker C, Heidenreich A, Nilsson S, Shore N. Current approaches to incorporation of radium-223 in clinical practice. Prostate Cancer Prostatic Dis. 2018;21:37-47.

7. Humeniuk MS, Gupta RT, Healy P, McNamara M, Ramalingam $\mathrm{S}$, Harrison $\mathrm{M}$, et al. Platinum sensitivity in metastatic prostate cancer: does histology matter? Prostate Cancer Prostatic Dis. 2018;21:92-99.

8. Aggarwal R, Huang J, Alumkal JJ, Zhang L, Feng FY, Thomas $\mathrm{GV}$, et al. Clinical and genomic characterization of treatmentemergent small-cell neuroendocrine prostate cancer: a multiinstitutional prospective study. J Clin Oncol. 2018;36: 2492-503.

9. Li R, Ravizzini GC, Gorin MA, Maurer T, Eiber M, Cooperberg MR, et al. The use of PET/CT in prostate cancer. Prostate Cancer Prostatic Dis. 2018;21:4-21.

10. Keizman D, Fosboel MO, Reichegger H, Peer A, Rosenbaum E, Desax MC, et al. Imaging response during therapy with radium223 for castration-resistant prostate cancer with bone metastasesanalysis of an international multicenter database. Prostate Cancer Prostatic Dis. 2017;20:289-93.

11. Calopedos RJS, Chalasani V, Asher R, Emmett L, Woo HH. Lutetium-177-labelled anti-prostate-specific membrane antigen antibody and ligands for the treatment of metastatic castrateresistant prostate cancer: a systematic review and meta-analysis. Prostate Cancer Prostatic Dis. 2017;20:352-60. 